Research Report

\title{
Excitotoxicity induced by kainic acid provokes glycogen synthase kinase-3 truncation in the hippocampus
}

\author{
Jerónimo Jurado-Arjona ${ }^{a, b}$, Paloma Goñi-Oliver ${ }^{a, 1}$, Lucía Rodríguez-Prada $^{a}$, \\ Tobias Engel ${ }^{c}$, D.C. Henshall ${ }^{c}$, Jesús Ávila ${ }^{a, b}$, Félix Hernández ${ }^{a, b, *}$ \\ aCentro de Biología Molecular "Severo Ochoa", CSIC/UAM, Universidad Autónoma de Madrid, Cantoblanco, \\ 28049 Madrid, Spain \\ ${ }^{\mathrm{b}}$ CIBERNED, Centro de Investigación Biomédica en Red de Enfermedades Neurodegenerativas, 28031 Madrid, Spain \\ ${ }^{\mathrm{c} D e p a r t m e n t}$ of Physiology and Medical Physics, Royal College of Surgeons in Ireland, 123 St. Stephen's Green, Dublin 2, \\ Ireland
}

\section{A R T I C L E I N F O}

\section{Article history:}

Accepted 6 March 2015

Available online 14 March 2015

\section{Keywords:}

Calpain

Glycogen synthase kinase-3 (GSK3)

Kainic acid (KA)

Excitotoxicity

Hippocampus

Proteolysis

\begin{abstract}
A B S T R A C T
In neuronal cultures, glycogen synthase kinase 3(GSK3) is truncated at the N-terminal end by calpain downstream of activated glutamate receptors. However, the in vivo biological significance of that truncation has not been explored. In an attempt to elucidate if GSK3 truncation has a pathophysiological relevance, we have used intraperitoneal injections of kainic acid (KA) in rats and intra-amygdala KA microinjections in mice as in vivo models of excitotoxicity. Spectrin cleavage analyzed by immunohistochemistry was observed in the CA1 hippocampal field in KA-intraperitoneal treated rats while the CA3 region was the hippocampal area affected after intra-amygdala KA microinjections. GSK3 $\beta$ immunofluorescence did not colocalize with truncated spectrin in both treatments using an antibody that recognize the N-terminal end of GSK3 $\beta$. Thus, those neurons which are spectrinpositive do not show GSK3 $\beta$ immunolabelling. To study GSK3 $\beta$ truncation in vitro, we exposed organotypic hippocampal slices and cultured cortical neurons to KA leading to the truncation of GSK3 and we found that truncation was blocked by the calpain inhibitor calpeptin. These data suggest a relationship between $\mathrm{N}$-terminal GSK3 $\beta$ truncation and excitotoxicity. Overall, our data reinforces the important relationship between glutamate receptors and GSK3 and their role in neurodegenerative processes in which excitotoxicity is involved.
\end{abstract}

(c) 2015 Elsevier B.V. All rights reserved.

\footnotetext{
*Corresponding author at: Centro de Biología Molecular "Severo Ochoa", CSIC/UAM, Universidad Autónoma de Madrid, Cantoblanco, 28049 Madrid, Spain. Fax: +34 911964420.

E-mail address: fhernandez@cbm.csic.es (F. Hernández).

${ }^{1}$ Present address: NeuroCure and Institute of Biochemistry, Charité-Universitätsmedizin-Berlin, Berlin, Germany.
} 


\section{Introduction}

GSK3 (Glycogen synthase kinase-3) is a kinase with two isoforms in mammals, GSK3 $\alpha$ with a molecular weight of $51 \mathrm{kDa}$ and GSK3 $\beta$ with a molecular weight of $47 \mathrm{kDa}$ (Woodgett, 1990). GSK3 can phosphorylate a variety of substrates including cytoskeletal proteins, transcription factors and metabolic regulators. Thus, GSK3 plays important roles in embryonic development, cell differentiation, microtubule dynamics, cell cycle division, cell adhesion, glucose metabolism and apoptosis. Deregulation of GSK3 activity is believed to play a key role in the pathogenesis of chronic central nervous system disorders such as Alzheimer disease $(\mathrm{AD})$, bipolar disorder and Huntington disease, as well as in metabolic disorders such as type II diabetes (Beurel et al., 2014; Hernandez and Avila, 2008; Jope and Johnson, 2004).

GSK3 is regulated by different mechanisms. One of these mechanisms is phosphorylation. Phosphorylation at Tyr-279/216 of GSK3 $\alpha / \beta$ is necessary for its activation (Hughes et al., 1993). In contrast, inactivation of GSK3 $\alpha / \beta$ is achieved by phosphorylation at Ser21/9, via a mechanism that involves the binding of a small loop containing the phosphorylated serine back to the substratebinding site on the enzyme (for a review see (Beurel et al., 2014). Another important mechanism controlling GSK3 function is through sequestration, as subcellular localization of GSK3 regulates substrates accessibility. GSK3 is observed in cytosol, mitochondria and nucleus, and also found enriched in specific neuronal compartments such as dendritic spines (Bijur and Jope, 2003; Jope and Johnson, 2004; Peineau et al., 2007). Therefore, restricting GSK3 to specific compartments may affect responsiveness to different signals. On the other hand, GSK3 can be regulated through binding to other proteins in several multiprotein complexes. The most studied pathway involving GSK3 complex formation is regulated by Wnt (Beurel et al., 2014).

Recently, a novel mechanism of regulation has been described: cleavage of GSK3 by calpain (Goni-Oliver et al., 2007; Ma et al., 2012). Cultured cortical neurons exposed to ionomycin, glutamate or N-methyl-D-aspartate (NMDA) led to the truncation of GSK3 (Goni-Oliver et al., 2007; Ma et al., 2009) as well as hippocampal cells exposed to 3-nitropropionic acid (Crespo-Biel et al., 2010b). However, the in vivo generation of that truncation has not been analyzed. In an attempt to elucidate if that truncation has a pathophysiological relevance, we have used two in vivo models of excitotoxicity employing intra-peritoneal kainic acid (KA)-injections in rats and intra-amygdala KA microinjections in mice. The work presented here demonstrates that in excitotoxicity conditions GSK3 suffers a process of proteolysis.

\section{Results}

\subsection{Characterization of Nterminal-GSK3 $\beta$ antibody}

To analyze GSK3 $\beta$ truncation in biological samples, we first characterized an antibody which is able to recognize full-length GSK3 $\beta$ but unable to recognize N-terminal truncated form. The anti-Nterminal-GSK3 $\beta$ monoclonal antibody was produced by immunizing animals with a synthetic peptide corresponding to the sequence of human GSK3 $\beta$ around aminoacid 22 (Cell Signaling, reference 9315). COS-7 cells were transfected with rat
Wt-GSK3 $\beta, \Delta$ C-GSK3 $\beta$ (aminoacids 1-384) and $\Delta$ N-GSK3 $\beta$ (aminoacids 39-420) constructs and extracts tested with several GSK3 antibodies. Fig. 1 shows as anti-Nterminal-GSK3 $\beta$ antibody recognizes full-length kinase and Cterminal-GSK3 $\beta$ truncated form, but does not recognize N-terminal truncated GSK3 $\beta$.

\subsection{Kainic acid induces GSK3 truncation in the hippocampus of rats and mice}

We first analyzed hippocampal samples by western blotting with anti-GSK3 antibody to determine whether GSK3 truncation was taking place after KA-i.p. treatment in rats. Upon treatment, additional GSK3 immunoreactive protein bands appeared at a lower molecular weight, reaching a maximum at $24 \mathrm{~h}$ (Fig. 2). These bands were only observed in overexposed gels, suggesting that their concentration was low. These bands were not detected in extracts prepared from saline-treated rats. We have previously described similar fragments in primary neuronal cultures after NMDA activation and shown this to be mediated by calpain (Goni-Oliver et al., 2007). Accumulation of alpha II spectrin fragments is a well characterized process associated with calpain activation (Czogalla and Sikorski, 2005). Fig. 2 shows the generation of lower molecular weight spectrin fragments in KA-i.p. treated rats in a time dependent manner suggesting that

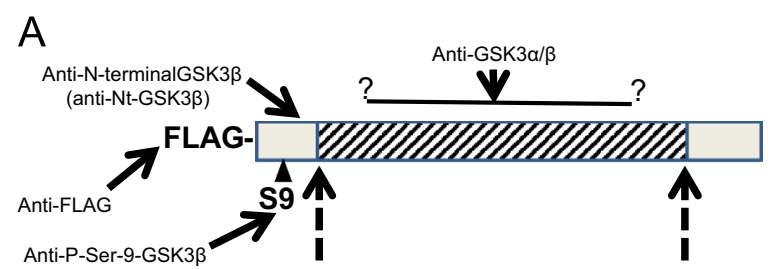

Thr-38-Thr-39

Ile-384-G/n-385

B

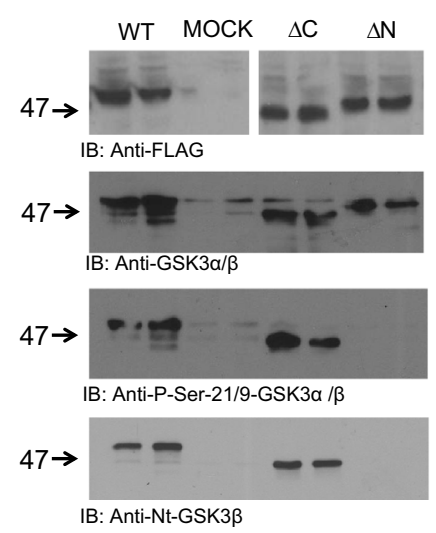

Fig. 1 - Characterization of Nterminal-GSK3 $\beta$ antibody. (A) Schematic diagrams of FLAG-GSK3 $\beta$ forms used showing the epitopes recognized by the GSK3 antibodies. The calpain cleavage sites are shown. (B) COS-7 cells were transfected with pcDNA3 (MOCK), rat Wt-GSK3 $\beta$ (WT), rat $\Delta \mathrm{C}-\mathrm{GSK} 3 \beta(\Delta \mathrm{C})$ and rat $\triangle \mathrm{N}$-GSK3 $\beta$ (constructs terminally FLAG-tagged) using Lipofectamine 2000. The samples were immunoblotted with the anti-FLAG antibody, anti-GSK3 $\alpha / \beta$ antibody, anti-pSer9/ 21-GSK3 $\alpha / \beta$ and anti-Nt-GSK3 $\beta$ antibody. The results show as Nt-GSK3 $\beta$ antibody recognizes full-length kinase and $\triangle$ C-GSK3 $\beta$, but does not recognize $\mathbf{N}$-terminal truncated form. 

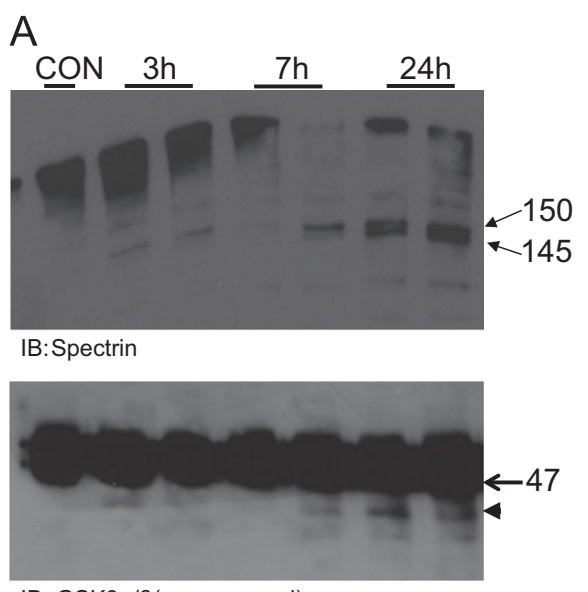

IB: GSK3a/ß(overexposed)

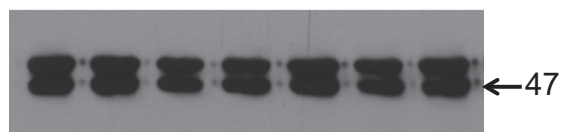

IB: GSK3a/ß

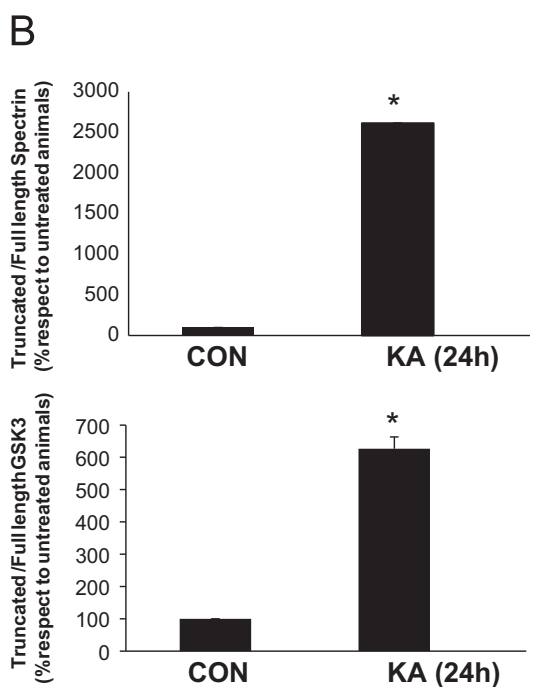

Fig. 2 - GSK3 truncation in response to i.p. KA-induced neurotoxicity. (A) Adult male wistar rats injected with KA or saline were sacrificed at various time points after injection. GSK3 and spectrin truncation was assessed by western blotting. Equal amounts of hippocampal extracts (15 $\mu \mathrm{g})$ were tested after 3, 7 or $24 \mathrm{~h}$ with anti-GSK3 $\alpha / \beta$ and anti-spectrin. Truncated GSK3forms were only seen in overexposed autoradiograms. A less exposed autoradiogram for anti-GSK3 $\alpha / \beta$ is also shown. Quantization of GSK3 and spectrin truncated forms after $24 \mathrm{~h}$ of treatment is shown in (B). Data are mean \pm SD from $n=3$ per group. * $p<0.05$ versus control samples. Molecular weights are shown in A. Arrow head indicates GSK3-truncated band quantified in $\mathbf{B}$.

spectrin truncation was taking place. The $145 \mathrm{kDa}$ band is a marker of calpain activation (Zhang et al., 2009). To analyze in which cells GSK3 truncation occurred we used the previously characterized antibody which recognizes the N-terminal end of GSK3 $\beta$ (Fig. 1). GSK3 $\beta$ is truncated at its $\mathrm{N}$ - and C-terminal ends at residues Thr-38-Thr-39 and Ile-384-Gln-385 (Ma et al., 2012). Thus, an antibody which recognizes the epitope around Ala22 does not recognize $\mathrm{N}$-terminal truncated fragment generated indicating that the enzyme is N-terminal truncated. To determine if both truncated proteins, spectrin and GSK3, colocalize we performed immunofluorescence studies to show if those neurons that are labeled by alpha II spectrin antibody are the same that display N-terminal GSK3 truncation. Double immunostaining with anti-Nterminal-GSK3 $\beta$ antibody and anti-spectrin showed that in control rats anti-Nterminal-GSK3 $\beta$ antibody labeled CA1, CA2 and CA3 areas without spectrin immunoreactivity (Fig. 3A). In $24 \mathrm{~h} \mathrm{KA-i.p.} \mathrm{treated} \mathrm{rats} \mathrm{anti-Nterminal-GSK3} \beta$ antibody signal disappeared and strong spectrin labeling can be observed in CA1 area (Fig. 3B). This region is the main hippocampal area affected in KA-i.p. treated rats (Araujo et al., 2008). Anti-Nterminal-GSK3 $\beta$ antibody labeled some cells in CA1 region, but these cells did not colocalize with cleaved spectrin labeled cells (Fig. 3C). These cells probably represent those cells where proteases are activated and where spectrin fragments accumulate. In good agreement, cells that were negative for spectrin were labeled by anti-Nterminal-GSK3 $\beta$ antibody (Fig. 3C).

In order to see if in another animal model of excitotoxicity produced the same GSK3 truncation we analyzed this response in mice. Taking into account that mouse strains vary significantly in their sensitivity to KA-induced neurodegeneration (Yang et al., 2005), we decided to analyze this response in mice that received an intra-amygdala injection of
KA a model of focally-evoked seizure-induced neuronal death using intra-amygdala KA microinjection and characterized by an acute hippocampal pathology (Mouri et al., 2008).

Intra-amygdala KA injection in mice results, after $4 \mathrm{~h}$, in damage that mainly affects the CA3 region (Mouri et al., 2008). When we analyzed samples from these mice, spectrin truncation was detected in the CA3 region and a decrease in GSK3 $\beta$ levels determined with the anti-Nterminal-GSK3 $\beta$ antibody was also observed only in that region (Fig. 4).

Thus, in two in vivo models of KA-induced hippocampal damage a decrease in Nterminal-GSK3 levels and an increase in spectrin truncation were observed. To analyze in more detail that effect we next carried out in vitro studies.

\subsection{Kainic acid induces GSK3 truncation in organotypic hippocampal cultures}

Given the role played by KA in GSK3 truncation in rodents, we explored whether kainate could be responsible for this GSK3 regulation in organotypic hippocampal cultures which reflect events happening in the hippocampus. Thus, we added KA $(50 \mu \mathrm{M})$ to these cultures and we analyzed those samples by western-blot. Fig. 5 shows that after $7 \mathrm{~h}$ of treatment, there was an increase not only in spectrin truncation but also in GSK3 truncation, and those proteolytic processes were inhibited by calpeptin $(10 \mu \mathrm{M})$, a calpain inhibitor.

\subsection{Kainic acid induces GSK3 truncation in neuronal primary cultures}

Then, we treated neuronal primary cultures with KA. Treatment of cultured cortical neurons with $\mathrm{KA}(50 \mu \mathrm{M})$ induced 


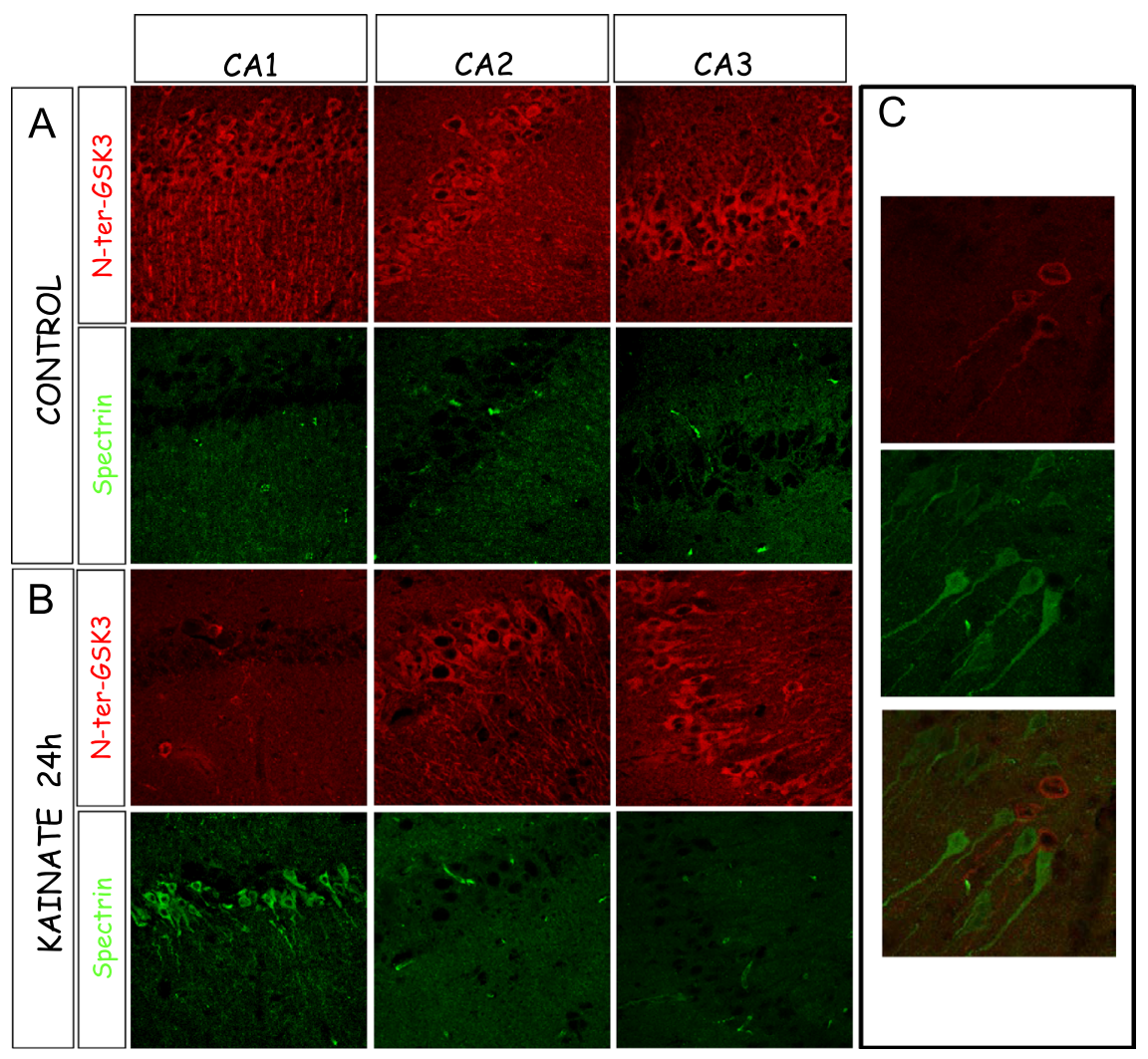

Fig. 3 - Spectrin and truncated GSK3 $\beta$ levels in rats treated i.p. with kainate. Representative confocal photomicrographs of hippocampal changes for spectrin (green) and Ntruncated GSK3 $\beta$ (red) in control rats (A) and KA treated rats (B) in CA1, CA2 and CA3 hippocampal regions. Images were taken from the same slice. CA2 and CA3 regions did not show differences among control and KA treated mice while CA1 area shows an increase in spectrin positive cells and a decrease in Nt-GSK3 $\beta$ signaling. (C) High magnification image of sample of KA treated rats of CA1 region. It should be noted that no colocalization of spectrin (green) and Nt-GSK3 $\beta$ (red) is observed.

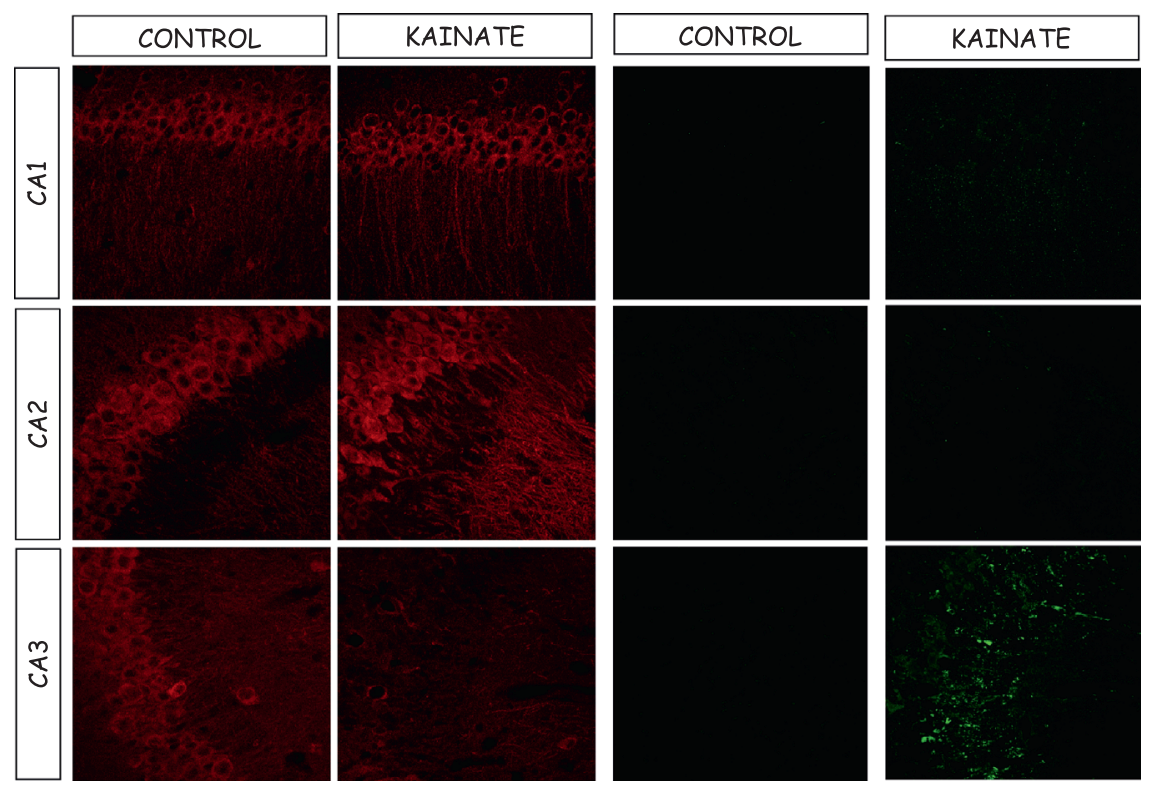

Fig. 4 - Spectrin and truncated GSK3 $\beta$ levels in intra-amygdala KA injected mice Representative confocal photomicrographs of hippocampal changes for spectrin (green) and Ntruncated GSK3 $\beta$ (red) antibodies in $4 \mathrm{~h}$ control and KA treated mice in hippocampal regions. CA1 and CA2 regions did not show differences among control and KA treated mice while CA3 area shows an increase in spectrin positive cells and a decrease in Nt-GSK3 $\beta$ signaling. 


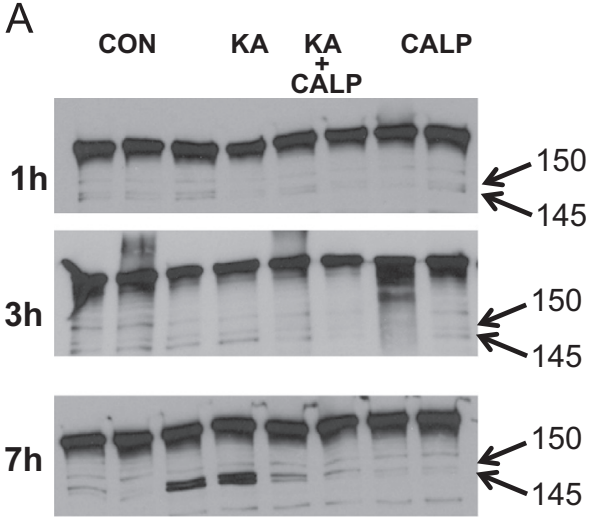

IB: Spectrin

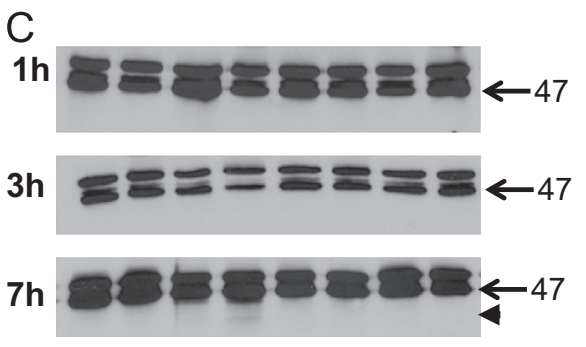

IB: GSK-3 $\alpha / \beta$
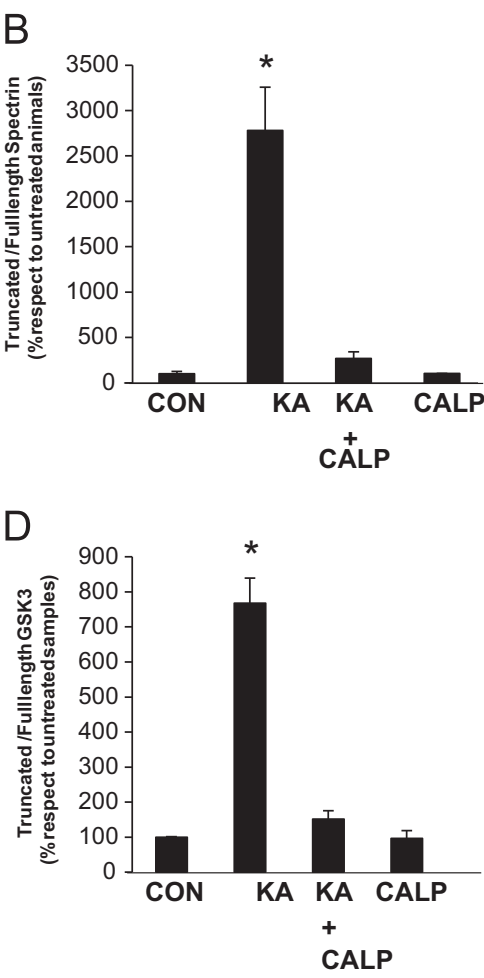

Fig. 5 - KA induces GSK3 and spectrin truncation in organotypic hippocampal slice cultures. Slices were treated with $50 \mu \mathrm{M}$ KA or vehicle (CON). Protein extracts $(15 \mu \mathrm{g})$ of slices treated with $\mathrm{KA}$ for 1,3 or $7 \mathrm{~h}$ in the presence or absence of $10 \mu \mathrm{M}$ calpeptin (CALP) were immunoblotted with the antibody that recognizes spectrin (A) and anti-GSK3 $\alpha / \beta$ antibody (C). Quantification of the truncated spectrin fragments (B) and the 40-kDa GSK3 truncated form (D) are shown. Bars, media \pm SD. ${ }^{*} p<0.05$ versus control. Arrow head indicates GSK3-truncated band quantified in D.

GSK3 truncation in a time-dependent manner (Fig. 6A) and was dose-dependent (EC50 $=1.81 \pm 0.95 \mu \mathrm{M})$ (Fig. 6B). The kainate effect was mediated by calpain as evidenced by the fact that calpeptin $(10 \mu \mathrm{M})$ prevented the formation of the $40 \mathrm{kDa}$ truncated form (Fig. 6C). The CDK5 regulator subunit p35, a well-known calpain substrate (Kusakawa et al., 2000), showed a similar pattern of protolysis, giving rise to the truncated p25 form (Fig. 6B). In addition, processing of full-length spectrin into break-down products denoted the activation of calpain. Thus, the EC50 for kainic acid induced alpha II spectrin truncation was $1.14 \pm 0.87 \mu \mathrm{M}$, similar to the EC50 obtained for GSK3-truncation. In addition, proteolysis of alpha II spectrin by calpain produces 150 and 145 breakdown products while caspase 3 produces a different $150 \mathrm{kDa}$ breakdown fragments (Zhang et al., 2009). Calpeptin inhibited the generation of the $145 \mathrm{kDa}$ fragment in cultured neurons while the $150 \mathrm{KDa}$ was highly decreased suggesting that alpha II spectrin is substrate of calpain but there is also some contribution by caspase 3. However, the calpain inhibitor calpeptin inhibited GSK3 truncation in cultured neurons and hippocampal slices suggesting that GSK3 is substrate of calpain only.

The antibody which recognize the epitope around Ala22 (Fig. 1) does not recognize any truncated fragment generated in KA-induced cultures suggesting that the $\mathrm{N}$-terminal end was removed by calpain (Fig. 6C). Indeed, the antibody that does not recognize the truncated form is able to detect the two full-length GSK3 $\beta$ isoforms generated by alternative splicing (Mukai et al., 2002). We next addressed whether the GSK3 truncation by calpain after stimulation of kainate receptors could be observed in the presence of an extracellular calcium chelator as EGTA (5 mM, Fig. 6D). Activation of the kainate receptors in the presence of extracellular EGTA led to inhibition of GSK3 proteolysis as was previously described for NMDA receptors (Goni-Oliver et al., 2009). These data suggest that extracellular calcium influx into cortical neurons caused by kainate receptors increase GSK3 truncation through calpain activation.

\section{Discussion}

Altered calcium homeostasis along with activation of calpain contributes to neuronal degeneration by cleavage of specific substrates that have crucial roles in neuronal survival (Camins et al., 2006; Huang and Wang, 2001; Patrick et al., 1999), including protein kinases (Goll et al., 2003). Previous studies have shown that GSK3 can undergo a limited proteolysis in cultured neurons (Crespo-Biel et al., 2010b; Czogalla and Sikorski, 2005; Feng et al., 2013; Goni-Oliver et al., 2007; Ma et al., 2012, 2009). However, it has been unclear whether this truncation of GSK3 occurs in vivo. In the present study we provide in vivo evidence that during KA-induced neuroexcitotoxicity, GSK3 is proteolytically truncated by calpain. In 

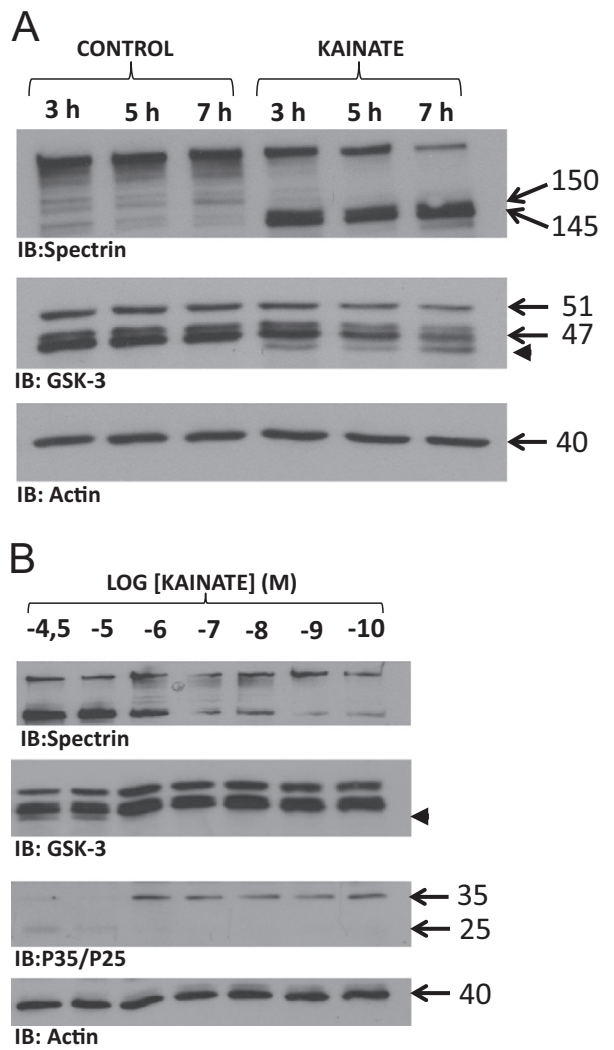

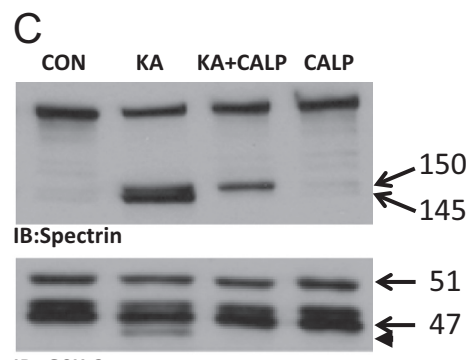

IB: GSK-3

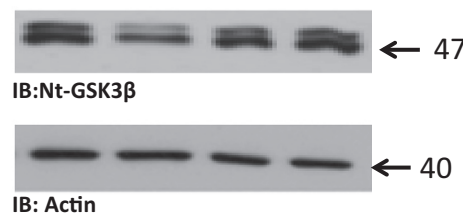

D

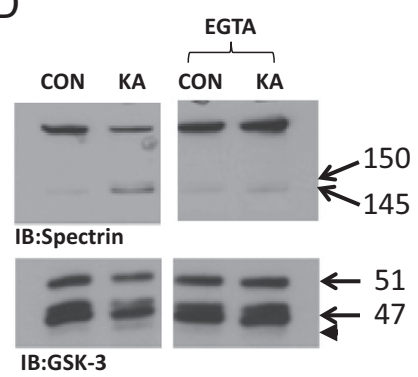

Fig. 6 - Proteolysis of GSK3 in cortical neurons treated with kainate. (A) Cortical neurons (DIV12) were treated with KA (50 $\mu$ M) or vehicle for 3, 5 or $7 \mathrm{~h}$. The samples were immunoblotted with the antibody that recognizes spectrin and the anti-GSK3 $\alpha / \beta$ antibody. The generation of a $40-\mathrm{kDa}$ GSK3 truncated form as well as the truncated spectrin fragments (150 and $145 \mathrm{kDa})$ is shown by the arrows. (B) Cortical neurons were treated with growing concentrations of KA for $5 \mathrm{~h}$. The samples were immunoblotted with the anti-GSK3 $\alpha / \beta$ antibody. The generation of a $40-\mathrm{kDa}$ GSK3 truncated form is shown by the head arrow. The same samples were also immunoblotted with the anti-p35/p25 antibody and the antibody that recognizes spectrin. (C) Neurons were treated with $50 \mu \mathrm{M} \mathrm{KA}$ in the presence or absence of the calpain inhibitor calpeptin ( $50 \mu \mathrm{M}, \mathrm{CALP})$ for $5 \mathrm{~h}$ and immunobloted with anti-spectrin, anti-GSK3 $\alpha / \beta$ antibody and the anti-Nterminal-GSK3 $\beta$ antibodies. Arrows show the 150 and $145 \mathrm{kDa}$ of spectrin fragments as well as truncated GSK3 fragment (D) Extracellular EGTA (5 mM) inhibits KA-induced truncation $(50 \mu \mathrm{M}, 5 \mathrm{~h}$ treatment). Arrow head indicates GSK3-truncated band.

these conditions we first demonstrated GSK3 truncation in the hippocampus in two animal models. Then, by using oganotypic hippocampal cultures and neuronal primary cultures we demonstrated that this is mediated by calpain.

In rats, systemic $\mathrm{KA}$ is a common model to study neuronal excitotoxicity. That model produce extensive damage, being the CA1 area the hippocampal region more affected (Araujo et al., 2008). In addition, and taking into account that mouse strains vary significantly in their sensitivity to KA-i.p. induced neurodegeneration (Yang et al., 2005), intra-amygdala injection of KA is a well characterized model of neuronal death in mice, being the CA3 hippocampal region the area predominantly damaged (Mouri et al., 2008). In both animal models of excitotoxicity we observed a decrease in Nterminal GSK-3 labeling suggesting that KA-induced neurodegeneration provokes GSK3 truncation in vivo and more important in those areas that are mainly affected in both models.

Proteases of the calpain and caspase families are involved in neurodegeneration mediated by calcium influx. Proteolysis of alpha II Spectrin by calpain produces 150 and $145 \mathrm{kDa}$ breakdown products while caspase 3 produces a different
$150 \mathrm{kDa}$ fragments (Zhang et al., 2009). In organotypic cultures as well as in cultured neurons, calpeptin inhibited the generation of the $145 \mathrm{kDa}$ fragment while the $150 \mathrm{KDa}$ was highly decreased suggesting that alpha II Spectrin is substrate of calpain but also caspase 3. However, the calpain inhibitor calpeptin inhibits GSK3 truncation in cultured neurons and hippocampal slices suggesting that GSK3 is substrate only of calpain. In fact, it has been reported that the caspase-3 inhibitor Z-VAD-FMK does not prevent the generation of GSK3 truncated fragments in rat cerebellar granule neurons (Ma et al., 2012). In addition, in the same KA injected rat model, caspases are not involved in GSK3 decreased levels (Araujo et al., 2008).

$\mathrm{N}$-terminal truncation eliminates the regulatory domain that inhibits the catalytic activity (Goni-Oliver et al., 2007; Ma et al., 2012). Thus, the present results seem to propose that during excitotoxicity processes there is an increase in GSK3 activity. We have not analyzed that increase; however, our results do allow us to state that during the excitotoxic process total GSK3 level decreased in cultured neurons. Interestingly, the $40 \mathrm{kDa}$ truncated form does not accumulate, suggesting that when 
GSK3 is truncated by calpain, a short lived product is formed that is rapidly degraded. What is the physiological significance of our results? It is interesting to note that lithium has a neuroprotective effect in KA-treated rats (Busceti et al., 2007; Crespo-Biel et al., 2010a). However, despite the neuroprotective effect of GSK3 inhibition, constitutive knock-out mice show embryonic lethality (Hoeflich et al., 2000). In this regard, pharmacological inhibition of GSK3 in cell lines has also been shown to facilitate apoptosis (Beurel and Jope, 2006) (Beyaert et al., 1989). Furthermore, transgenic mice with expression of a dominant negative form of GSK3 $\beta$ showed that a reduction in GSK3 activity induces apoptosis in neurons (Gomez-Sintes et al., 2007). Our data seem to suggest that in excitotoxic situations, likely due to an increase in cytosolic calcium and calpain activation, those cells that are spectrin positives are suffering a decrease in GSK3 levels. Interestingly, an overexpression of GSK3 $\beta$ truncated forms in rat cerebellar granule neurons generated by calpain induces apoptosis as well (Ma et al., 2012).

A special feature of our work is the characterization of an antibody which is able to recognize full-length GSK3 $\beta$ but unable to recognize $\mathrm{N}$-terminal truncated form. Calpain cleaves GSK3 $\beta$ in vitro not only at the $\mathrm{N}$-terminal (at residues Thr-38-Thr-39) but also at the C-terminal end (at residues Ile384-Gln-385) (Ma et al., 2012). However, our results suggest that C-terminal truncation does not take place in KA treated neurons. The anti-Nterminal-GSK3 $\beta$ monoclonal antibody used is not able to recognize any band of lower molecular weight (Fig. 5C), although it recognized the isoform C-terminal truncated (Fig. 1) and the full-length isoforms of GSK3ß (Fig. 5C) (Mukai et al., 2002). However, it cannot be discard that C-truncated form could be a short life fragment or that, as has been described previously, high levels of Ser389 phosphorylation of GSK3 cold inhibits C-terminal cleavage (Ma et al., 2012).

\section{Conclusions}

To summarize, the present results have clear implications in all the transduction pathways in which GSK3 is involved. Taking into account that calpains induce p35 truncation and that calpain also induces GSK3 truncation, it is possible that the deregulation of calcium homeostasis may contribute to neuropathology through the calpain/CDK5/GSK3 system. Thus, our data support the view that calpains may provide a link between both kinases and provide cues for the understanding of GSK3 involvement in neurodegenerative processes where excitotoxicity conditions are present.

\section{Experimental procedures}

\subsection{Animals and tissue processing.}

\subsubsection{Animal care}

Rats (Wistar, 2 months) and mice (C57BL/6J, 1 month) were treated following the guidelines of Council of Europe Convention ETS123, revised as indicated in the Directive 86/609/ EEC. In addition all protocols were approved by the institutional animal care and use committee. Animals were bred at the Centro de Biología Molecular "Severo Ochoa" (Madrid, Spain) and were kept on a normal light-dark cycle (12 h light/12 h dark), with free access to food and water.

\subsubsection{Kainic acid treatments}

KA was dissolved in saline and rats were injected intraperitoneally at $10 \mathrm{mg} / \mathrm{kg}$ body weight and monitored for responsiveness to KA. Intra-amygdala KA injections were performed according to a previously described technique and killed $4 \mathrm{~h}$ later (Mouri et al., 2008).

\subsubsection{Tissue processing}

Animals were sacrificed using $\mathrm{CO}_{2}$ inhalation and the brains were removed, post-fixed overnight in $4 \%$ PFA and $30 \mu \mathrm{m}$ sagittal sections were obtained on a cryostat.

\subsection{Primary cultures}

Primary cultures of mouse cortical neurons were prepared according to modifications of established procedures (Hernandez et al., 2004). Briefly, mouse pups were sacrificed at postnatal day 1 . Cortical tissue was then dissected and dissociated individually from each pup with the papain dissociation system (Worthington Biochemical Corp.). Cortical neurons were maintained in neurobasal medium (Invitrogen) supplemented with 1\% B-27, 5\% fetal calf serum, $0.5 \mathrm{mM}$ glutamine, 100 units $/ \mathrm{ml}$ penicillin, and $100 \mathrm{mg} / \mathrm{ml}$ streptomycin and grown on $3 \mu \mathrm{g} / \mathrm{ml}$ laminin (Sigma) and $10 \mu \mathrm{g} / \mathrm{ml}$ poly-L-lysine-coated cover plates. The cells were incubated in $95 \%$ air, $5 \% \mathrm{CO}_{2}$ in a humidified incubator at $37{ }^{\circ} \mathrm{C}$. Cytosine arabinoside $(2.5 \mu \mathrm{M})$ was added to cultures on the second day after seeding (DIV3) to inhibit the proliferation of nonneuronal cells. Cells were used for experiments at DIV12.

\subsection{Transfection of COS-7 cell}

COS-7 transfection studies were carried out using constructs kindly provided by Dr Mingtao Li, Sun Yat-sen University, China (Ma et al., 2012). The rat Wt-GSK3 $\beta, \Delta C-G S K 3 \beta$ (aminoacids 1-384) and $\Delta \mathrm{N}-\mathrm{GSK} 3 \beta$ (aminoacids 39-420) constructs are $\mathrm{N}$-terminally FLAG-tagged and cloned into pcDNA3.1 vector. COS-7 cells were transfected using Lipofectamine 2000 (Invitrogen) according to the manufacturer's instructions.

\subsection{Organotypic hippocampal slice cultures}

Hippocampal slice cultures were prepared from 1 mouth-old C57BL/6J mice. Animals were decapitated; the brains were removed and transferred to cold Minimal Essential Medium (MEM). A tissue chopper was used to cut hippocampal slices $(400 \mu \mathrm{m})$, which were placed on porous $0.4 \mathrm{~mm}$ membranes (Millipore). The membranes were then transferred to culture plates covered with culture medium (Neurobasal Medium supplemented $5 \%$ horse serum and B27). The culture medium was changed two times per week. Slice cultures were used for experiments after 7 days in vitro.

\subsection{Western blot analysis}

Brains were quickly dissected on an ice-cold plate. Hippocampus was isolated and extracts for western blot analysis 
were prepared by homogenizing in ice-cold extraction buffer consisting of $50 \mathrm{mM}$ Tris $\mathrm{HCl}, \mathrm{pH} 7.4,150 \mathrm{mM} \mathrm{NaCl}, 1 \% \mathrm{NP}-40$, $1 \mathrm{mM}$ sodium orthovanadate, $1 \mathrm{mM}$ EDTA, a protease inhibitor cocktail (Roche) and $1 \mu \mathrm{M}$ Okadaic acid (phosphatase inhibitor). The samples were homogenized and protein content was determined by Bradford. Thirty micrograms of total protein were electrophoresed on 10\% SDS-polyacrylamide gel and transferred to a nitrocellulose membrane (Schleicher and Schuell, Keene, NH). The experiments were performed using the following primary monoclonal antibodies: anti-GSK3 $\alpha / \beta$ (1/1000, BD Transduction Laboratories), anti-p21/9- GSK3 $\alpha / \beta$ (1/500, Cell Signaling), anti-Nterminal-GSK3 $\beta$ (1/1000, reference 9315 Cell Signaling), anti-spectrin (1/1000, Chemicon International), anti-p35/25 (1/500, Santa Cruz), anti-FLAG (1/5000, Sigma) and anti-actin (1/5000, Sigma). Filters were incubated with the antibody at $4{ }^{\circ} \mathrm{C}$ overnight in $5 \%$ nonfat dried milk. Secondary goat anti-mouse and anti-rabbit antibodies (1/1000; Invitrogen, San Diego, CA) and ECL detection reagents (Amersham Biosciences, Arlington Heights, IL) were used for immunodetection. Quantification was performed by densitometric scanning. The densitometry values were obtained in the linear range of detection with these antibodies. These values were normalized with respect to the values obtained with an anti- $\beta$-actin antibody to correct for total protein content.

\subsection{Immunostaining}

For immunofluorescence, immunostaining was carried out following a standard procedure. Sections were incubated with the primary antibody overnight at $4{ }^{\circ} \mathrm{C}$ in a $\mathrm{PB}$ solution containing BSA $1 \%$ and TritonX-100 1\%. Primary antibodies were: anti-GSK3 $\alpha \beta$ (1/100), anti-Nterminal-GSK3 $\beta$ (1/100) and anti-spectrin (1/100). After washing with blocking solution 3 times, sections were incubated with donkey Alexaconjugated secondary antibodies (anti-rabbit, anti-mouse Alexa-Fluor $488 / 555$-conjugated) overnight at $4{ }^{\circ} \mathrm{C}(1: 1,000)$ (Molecular Probes, Millipore). Finally, after washing with phosphate buffer solution, sections were incubated with DAPI $(1 / 2,000)$ (Calbiochem) for $10 \mathrm{~min}$. Images are single sections of a z-series acquiring each channel in a sequential mode using an inverted Zeiss LSM710 confocal microscope with a 63X/1.40 Plan-Apochromatic objective.

\subsection{Statistical analysis}

The SPSS 17.0.1 softwaSigmaPlot 12.5 software was used for EC50 experiments were carried out in triplicate. Student $t$ test was used for single comparisons.

\section{Acknowledgments}

Our laboratory is funded by grants from Ministerio de Educación y Ciencia (SAF2010-15525 and BUF2013-40664-P) and Comunidad de Madrid (S2010/BMD-2331). We also acknowledge institutional support from Fundación Ramón Areces. The authors would also like to thank the Health Research Board Ireland for support (PD/2009/31 to TE and HRA_POR/ 2011/41 to DCH).

\section{R E F E R E N C E S}

Araujo, I.M., Gil, J.M., Carreira, B.P., Mohapel, P., Petersen, A., Pinheiro, P.S., Soulet, D., Bahr, B.A., Brundin, P., Carvalho, C.M., 2008. Calpain activation is involved in early caspaseindependent neurodegeneration in the hippocampus following status epilepticus. J. Neurochem. 105, 666-676.

Beurel, E., Jope, R.S., 2006. The paradoxical pro- and antiapoptotic actions of GSK3 in the intrinsic and extrinsic apoptosis signaling pathways. Prog. Neurobiol. 79, 173-189.

Beurel, E., Grieco, S.F., Jope, R.S., 2014. Glycogen synthase kinase-3 (GSK3): regulation, actions, and diseases. Pharmacol. Ther..

Beyaert, R., Vanhaesebroeck, B., Suffys, P., Van Roy, F., Fiers, W., 1989. Lithium chloride potentiates tumor necrosis factormediated cytotoxicity in vitro and in vivo. Proc. Natl. Acad. Sci. USA 86, 9494-9498.

Bijur, G.N., Jope, R.S., 2003. Glycogen synthase kinase-3 beta is highly activated in nuclei and mitochondria. Neuroreport 14, 2415-2419.

Busceti, C.L., Biagioni, F., Aronica, E., Riozzi, B., Storto, M., Battaglia, G., Giorgi, F.S., Gradini, R., Fornai, F., Caricasole, A., Nicoletti, F., Bruno, V., 2007. Induction of the Wnt inhibitor, Dickkopf-1, is associated with neurodegeneration related to temporal lobe epilepsy. Epilepsia 48, 694-705.

Camins, A., Verdaguer, E., Folch, J., Pallas, M., 2006. Involvement of calpain activation in neurodegenerative processes. CNS Drug Rev. 12, 135-148.

Crespo-Biel, N., Camins, A., Canudas, A.M., Pallas, M., 2010a. Kainate-induced toxicity in the hippocampus: potential role of lithium. Bipolar Disord. 12, 425-436.

Crespo-Biel, N., Camins, A., Gutierrez-Cuesta, J., Melchiorri, D., Nicoletti, F., Pallas, M., Canudas, A.M., 2010b. Regulation of GSK-3beta by calpain in the 3-nitropropionic acid model. Hippocampus 20, 962-970.

Czogalla, A., Sikorski, A.F., 2005. Spectrin and calpain: a 'target' and a 'sniper' in the pathology of neuronal cells. Cell. Mol. Life Sci. 62, 1913-1924.

Feng, Y., Xia, Y., Yu, G., Shu, X., Ge, H., Zeng, K., Wang, J., Wang, X., 2013. Cleavage of GSK-3beta by calpain counteracts the inhibitory effect of Ser9 phosphorylation on GSK-3beta activity induced by $\mathrm{H}_{2} \mathrm{O}_{2}$. J. Neurochem. 126, 234-242.

Goll, D.E., Thompson, V.F., Li, H., Wei, W., Cong, J., 2003. The calpain system. Physiol. Rev. 83, 731-801.

Gomez-Sintes, R., Hernandez, F., Bortolozzi, A., Artigas, F., Avila, J., Zaratin, P., Gotteland, J.P., Lucas, J.J., 2007. Neuronal apoptosis and reversible motor deficit in dominant-negative GSK-3 conditional transgenic mice. EMBO J. 26, 2743-2754.

Goni-Oliver, P., Lucas, J.J., Avila, J., Hernandez, F., 2007. N-terminal cleavage of GSK-3 by calpain: a new form of GSK-3 regulation. J. Biol. Chem. 282, 22406-22413.

Goni-Oliver, P., Avila, J., Hernandez, F., 2009. Memantine inhibits calpain-mediated truncation of GSK-3 induced by NMDA: implications in Alzheimer's disease. J. Alzheimers Dis. 18, 843-848.

Hernandez, F., Perez, M., Lucas, J.J., Mata, A.M., Bhat, R., Avila, J., 2004. Glycogen synthase kinase-3 plays a crucial role in tau exon 10 splicing and intranuclear distribution of SC35. Implications for Alzheimer's disease. J. Biol. Chem. 279, 3801-3806.

Hernandez, F., Avila, J., 2008. The role of glycogen synthase kinase 3 in the early stages of Alzheimers' disease. FEBS Lett. 582, 3848-3854.

Hoeflich, K.P., Luo, J., Rubie, E.A., Tsao, M.S., Jin, O., Woodgett, J.R., 2000. Requirement for glycogen synthase kinase-3beta in cell survival and NF-kappaB activation. Nature 406, 86-90.

Huang, Y., Wang, K.K., 2001. The calpain family and human disease. Trends Mol. Med. 7, 355-362. 
Hughes, K., Nikolakaki, E., Plyte, S.E., Totty, N.F., Woodgett, J.R., 1993. Modulation of the glycogen synthase kinase-3 family by tyrosine phosphorylation. EMBO J. 12, 803-808.

Jope, R.S., Johnson, G.V., 2004. The glamour and gloom of glycogen synthase kinase-3. Trends Biochem. Sci. 29, 95-102.

Kusakawa, G., Saito, T., Onuki, R., Ishiguro, K., Kishimoto, T., Hisanaga, S., 2000. Calpain-dependent proteolytic cleavage of the p35 cyclin-dependent kinase 5 activator to p25. J. Biol. Chem. 275, 17166-17172.

Ma, S., Liu, S., Huang, Q., Xie, B., Lai, B., Wang, C., Song, B., Li, M., 2012. Site-specific phosphorylation protects glycogen synthase kinase-3beta from calpain-mediated truncation of its N and C termini. J. Biol. Chem. 287, 22521-22532.

Ma, T., Zhao, Y., Kwak, Y.D., Yang, Z., Thompson, R., Luo, Z., Xu, H., Liao, F.F., 2009. Statin's excitoprotection is mediated by sAPP and the subsequent attenuation of calpain-induced truncation events, likely via rho-ROCK signaling. J. Neurosci. 29, 11226-11236.

Mouri, G., Jimenez-Mateos, E., Engel, T., Dunleavy, M., Hatazaki, S., Paucard, A., Matsushima, S., Taki, W., Henshall, D.C., 2008. Unilateral hippocampal CA3-predominant damage and short latency epileptogenesis after intra-amygdala microinjection of kainic acid in mice. Brain Res. 1213, 140-151.

Mukai, F., Ishiguro, K., Sano, Y., Fujita, S.C., 2002. Alternative splicing isoform of tau protein kinase I/glycogen synthase kinase 3beta. J. Neurochem. 81, 1073-1083.
Patrick, G.N., Zukerberg, L., Nikolic, M., delaMonte, S., Dikkes, P., Tsai, L.H., 1999. Conversion of p35 to p25 deregulates Cdk5 activity and promotes neurodegeneration. Nature 402, 615-622.

Peineau, S., Taghibiglou, C., Bradley, C., Wong, T.P., Liu, L., Lu, J., Lo, E., Wu, D., Saule, E., Bouschet, T., Matthews, P., Isaac, J.T., Bortolotto, Z.A., Wang, Y.T., Collingridge, G.L., 2007. LTP inhibits LTD in the hippocampus via regulation of GSK3beta. Neuron 53, 703-717.

Woodgett, J.R., 1990. Molecular cloning and expression of glycogen synthase kinase-3/factor A. EMBO J. 9, 2431-2438.

Yang, J., Houk, B., Shah, J., Hauser, K.F., Luo, Y., Smith, G., Schauwecker, E., Barnes, G.N., 2005. Genetic background regulates semaphorin gene expression and epileptogenesis in mouse brain after kainic acid status epilepticus. Neuroscience 131, 853-869.

Zhang, Z., Larner, S.F., Liu, M.C., Zheng, W., Hayes, R.L., Wang, K. K., 2009. Multiple alphaII-spectrin breakdown products distinguish calpain and caspase dominated necrotic and apoptotic cell death pathways. Apoptosis 14, 1289-1298. 